\title{
Surrogacy of Time to Progression for Overall Survival in Advanced Hepatocellular Carcinoma Treated with Systemic Therapy: A Systematic Review and Meta-Analysis of Randomized Controlled Trials
}

\author{
Takeshi Terashima $^{a} \quad$ Tatsuya Yamashita $^{a}$ Tadashi Toyama ${ }^{b}$ Kuniaki Arai ${ }^{a}$ \\ Kazunori Kawaguchi ${ }^{a}$ Kazuya Kitamura ${ }^{a}$ Taro Yamashita ${ }^{a}$ Yoshio Sakai ${ }^{a}$ \\ Eishiro Mizukoshi $^{a} \quad$ Masao Honda $^{a}$ Shuichi Kaneko ${ }^{a}$ \\ aDepartment of Gastroenterology, Kanazawa University Hospital, Kanazawa, Japan; \\ ${ }^{b}$ Department of Nephrology, Kanazawa University Hospital, Kanazawa, Japan
}

\section{Keywords}

Time to progression - Overall survival - Surrogate endpoint · Hepatocellular carcinoma .

Clinical trials · Molecular targeted therapy

\begin{abstract}
Time to progression (TTP) is widely used as the endpoint in early-phase trials of advanced hepatocellular carcinoma (HCC). However, the relevance of using TTP as a surrogate marker for overall survival (OS) in pivotal trials remains uncertain. The PubMed database and ASCO Meeting Library were searched for reports of randomized controlled trials that investigated patients with advanced HCC, included data for both OS and TTP, and were launched between 2009 and 2016. The correlation between hazard ratios (HRs) for TTP and OS was determined using weighted linear regression. Correlations between median OS and TTP, and between median OS and postprogression survival (PPS), defined as the period obtained by subtracting the median TTP from the median OS, were also evaluated. The database search yielded 24 trials with 50 arms. Overall, TTP HR correlated with OS HR $(R=0.73)$; however, the coefficient in the regression equation was 0.48 . The correlation between median OS and median TTP was not so strong $(R=0.50)$, whereas the correlation between median OS and median PPS was strong $(R=0.78)$. In advanced HCC, the OS HR can be predicted from the TTP HR, which is useful when considering whether to proceed to a pivotal trial based on the results of earlyphase trials. TTP may be a better endpoint than OS for evaluating a novel agent in a pivotal trial, because an improvement in antitumor effect cannot fully reflect an improvement in OS due to the strong impact of PPS on OS.




\section{Liver Cancer}

\section{Introduction}

Overall survival (OS) has been considered the optimal and firm endpoint in clinical trials for cancer patients who cannot receive curative treatment, because the aim for such patients is prolongation of survival. However, it takes a long time to complete a clinical trial when OS is set as the primary endpoint, and an alternative endpoint, such as time to progression (TTP), is commonly used for early-phase trials to evaluate the treatment efficacy of a novel agent over a short time $[1,2]$. Although the objective response rate was also suggested as a representative indicator across various cancer patients, its suitability as a surrogate endpoint for an agent that does not act through shrinkage of the tumor is still debated $[3,4]$.

Hepatocellular carcinoma (HCC) is the sixth most common cancer and the third leading cause of cancer-related death worldwide [5]. Since sorafenib showed a survival benefit for patients with advanced HCC $[6,7]$, many novel agents have been tested in randomized phase III trials in first-line [8-14] and second-line [15-19] settings. Some agents have shown a significant antitumor effect in terms of prolongation of the TTP $[9,12,15,17,18]$; however, with the exception of regorafenib [20], none have been proven to prolong survival.

All trials to date have set OS as the primary endpoint, and the relevance of using TTP as a surrogate marker for OS remains unclear. We evaluated the correlation between TTP hazard ratio (HR) and OS HR based on a systematic review and meta-analysis of randomized controlled trials to clarify the value of improvement of TTP as a surrogate for improvement of OS. We also evaluated the correlation between median OS (mOS) and median TTP (mTTP) and between mOS and median postprogression survival (mPPS), which was defined as the period obtained by subtracting mTTP from mOS. This approach will clarify the applicability of TTP as a surrogate marker for OS and provide information that is useful for planning and interpreting clinical trials on patients with advanced HCC.

\section{Materials and Methods}

\section{Search and Selection of Literature}

We reviewed PubMed citations and the ASCO Meeting Library up to December 31, 2016. Keywords included in the search were "advanced hepatocellular carcinoma" and "randomized trial." The search was limited to reports of randomized controlled trials written in English. Furthermore, we also checked the Cochrane Library and ESMO Conference Platform to confirm the randomized controlled trials which should be included in this study. We reviewed each report including publications on the website and selected studies that compared two or more arms for treatment of advanced HCC with a medical agent.

Included in this study were randomized phase II or phase III trials that provided an analysis of intent-totreat data for OS HRs and either TTP HRs or progression-free survival (PFS) HRs of an experimental arm against a control arm. We excluded review articles, letters or commentaries, interim analyses, subgroup analyses of previously reported trials, or duplicate reports. Reports in which all or some patients were treated in an adjuvant setting or neoadjuvant setting - or with concomitant treatment procedures including resection, transplantation, transarterial chemoembolization, transarterial infusion chemotherapy, or radiation therapy in addition to systemic therapy - were also excluded. To avoid bias, two investigators (T. Terashima and K.A.) independently reviewed and abstracted the data from the reports, and another investigator (T. Yamashita) made the final decision if agreement was not reached between the first two investigators.

\section{Data Extraction}

For simplicity, PFS data were collectively referred to as TTP in the present analysis if TTP was not addressed, which is similar to an approach adopted in a recent report [21]. TTP and OS HRs, mTTP, and mOS were extracted from all reports that provided data. MPPS was defined as the period obtained by subtracting mTTP from mOS for each report. The survival data were converted into months; 1 month was considered to be 30.45 days in reports that described survival data in days. We also obtained the following information from each report: the number of arms, trial phase, publication status, treatment line, the type of control arm, and the number of patients in each arm. 


\section{Liver Cancer}

Table 1. Characteristics of the trials used in this study

\begin{tabular}{l|l}
\hline Liver Cancer 2019;8:130-139 \\
\hline DOI: 10.1159/000489505 & $\begin{array}{l}\text { @ 2018 S. Karger AG, Basel } \\
\text { www.karger.com/lic }\end{array}$ \\
\hline
\end{tabular}

\section{Data Analysis}

We summarized the survival data (TTP HR, OS HR, mOS, MTTP, and MPPS) as the median value for all treatment arms. The relationship between TTP HR and OS HR was estimated using weighted linear regression, with weights equal to the sample size of the arms from which the data were derived [22]. All reported $p$ values correspond to two-sided tests, with $p<0.05$ considered to be statistically significant. The strength of the association was assessed by using $R$, and we considered the correlation to be strong when $R$ was $\geq 0.7$. We also evaluated whether the relationship changed according to treatment line (first vs. second line), assessment of tumor progression (TTP vs. PFS), trial phase (randomized phase II vs. phase III), and publication status (published vs. unpublished trials). The relationship between mOS and either mTTP or mPPS was analyzed in the same way. The data analyses were performed using Stata 12.1 (College Station, TX, USA).

\section{Results}

\section{Characteristics of the Trials}

We identified 30 trials in the initial search of PubMed and ASCO Meeting Library citations, but excluded 6 trials because of lack of information about TTP HR and/or OS HR (see online suppl. Fig. 1; see www.karger.com/doi/10.1159/000489505 for all online suppl. material). The remaining 24 trials were included in the present study. The characteristics of the 24 trials, which included 50 arms and 9,556 patients with advanced HCC, are shown in Table 1 and the online supplementary Table. Sixteen trials were in the first-line setting and 8 trials were in the second-line setting. The number of patients in each arm ranged from 19 to 578 , with a median of 134.5 .

\section{Relationships between HRs for TTP and OS}

The TTP HRs of experimental arms against the control arm ranged from 0.5 to 1.4, with a median TTP HR of 0.915 , whereas the corresponding OS HR ranged from 0.44 to 1.3 , with a median OS HR of 0.918.

The OS HR of the 24 trials was plotted against the TTP HR (Fig. 1). Overall, TTP HR strongly correlated with OS HR $(R=0.73, p<0.001)$ based on weighted linear regression. 


\section{Liver \\ Cancer}

Fig. 1. Relationship between overall survival hazard ratio (OS HR) and time to progression/progression-free survival HR (TTP/ PFS HR) among all trials. Each trial is represented by a circle, with its size proportional to the number of patients. TTP/PFS HR was strongly correlated with OS HR $(R=0.73, p<0.001)$ on the basis of weighted linear regression $\left(^{*}\right)$.

Fig. 2. Relationship between overall survival hazard ratio (OS HR) and time to progression/progression-free survival HR (TTP/ PFS HR) among second-line trials (a) and first-line trials (b). Each trial is represented by a circle, with its size proportional to the number of patients. TTP/PFS HR was more strongly correlated with OS HR in a second-line setting $(R=0.91, p=0.002)$ than in a first-line $(R=0.77, p<0.001)$ setting on the basis of weighted linear regression $\left(^{*}\right)$.

\begin{tabular}{|c|c|}
\hline \multicolumn{2}{|l|}{ Liver Cancer 2019;8:130-139 } \\
\hline DOI: $10.1159 / 000489505$ & $\begin{array}{l}\text { (c) } 2018 \text { S. Karger AG, Basel } \\
\text { www.karger.com/lic }\end{array}$ \\
\hline
\end{tabular}

Terashima et al.: TTP and Endpoint in Advanced HCC
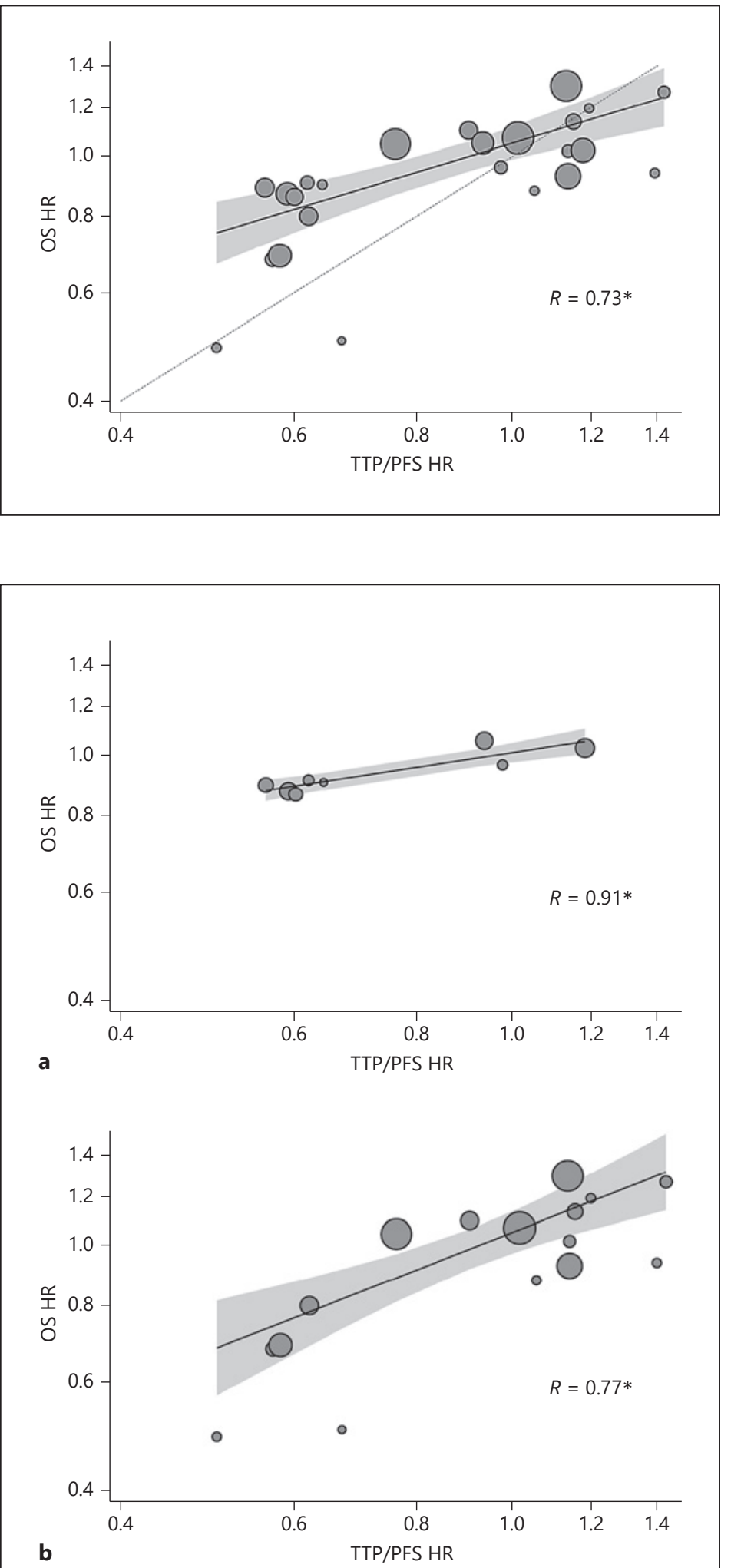

b

TTP/PFS HR 


\section{Liver \\ Cancer}

Fig. 3. Relationship between overall survival hazard ratio (OS $\mathrm{HR}$ ) and time to progression (TTP) HR among the trials in which tumor progression was assessed by TTP (a) and progression-free survival (PFS) (b). Each trial is represented by a circle, with its size proportional to the number of patients. TTP HR and PFS HR were consistently correlated with OS $\operatorname{HR}(R=0.72$ and $R=$ 0.70 , respectively) on the basis of weighted linear regression $\left.{ }^{*}\right)$.

\begin{tabular}{l|l}
\hline Liver Cancer 2019;8:130-139 \\
\hline DOI: 10.1159/000489505 & $\begin{array}{l}\text { @ 2018 S. Karger AG, Basel } \\
\text { www.karger.com/lic }\end{array}$ \\
\hline
\end{tabular}



However, the coefficient in the regression equation was not much greater than 0.48 , and the regression equation was $\log (\mathrm{OS} H \mathrm{HR})=0.48 \log (\mathrm{TTP} H \mathrm{HR})+0.050$. When trials were stratified by treatment line, TTP HR was more strongly correlated with OS HR in second-line $(R=0.91$, $p=0.002$ ) (Fig. 2a) than in first-line $(R=0.77, p<0.001)$ (Fig. 2b) settings. Corresponding tendencies were observed in the sensitivity analysis conducted to confirm the robustness of the above results among the reports in which tumor progression was assessed by TTP $(R=$ $0.70, p<0.001$ ) (Fig. 3a) and PFS ( $R=0.71, p=0.003$ ) (Fig. 3b), the phase III trials (see online suppl. Fig. 2A), and the published trials (see online suppl. Fig. 2B).

\section{Relationships between Median Times of OS and TTP or PPS}

mOS, mTTP, and mPPS for all arms was 4.2-13.7 months (median 8.6), 0.95-6.4 months (median 3.0), and 2.8-9.8 months (median 5.2), respectively. The proportion of mPPS to mOS for all arms was $40.7-86.6 \%$, with a median of $63.9 \%$.

mOS in the 50 arms was plotted against mPPS (Fig. 4a) and mTTP (Fig. 4b). The correlation between mOS and mTTP was not so strong $(R=0.50, p<0.001)$, whereas the correlation between mOS and mPPS was strong $(R=0.78, p<0.001)$, based on weighted linear regression. 
Fig. 4. Relationship between median overall survival (OS) and median time to progression (TTP) or progression-free survival (PFS) (a), and between median OS and median postprogression survival (PPS) (b), among all trials. Each trial is represented by a circle, with its size proportional to the number of patients. The correlation between median OS and median TTP or PFS was not strong $(R=0.50, p<0.001)$, whereas the correlation between median OS and median PPS was strong $(R=$ $0.78, p<0.001$ ) based on weighted linear regression $(*)$.

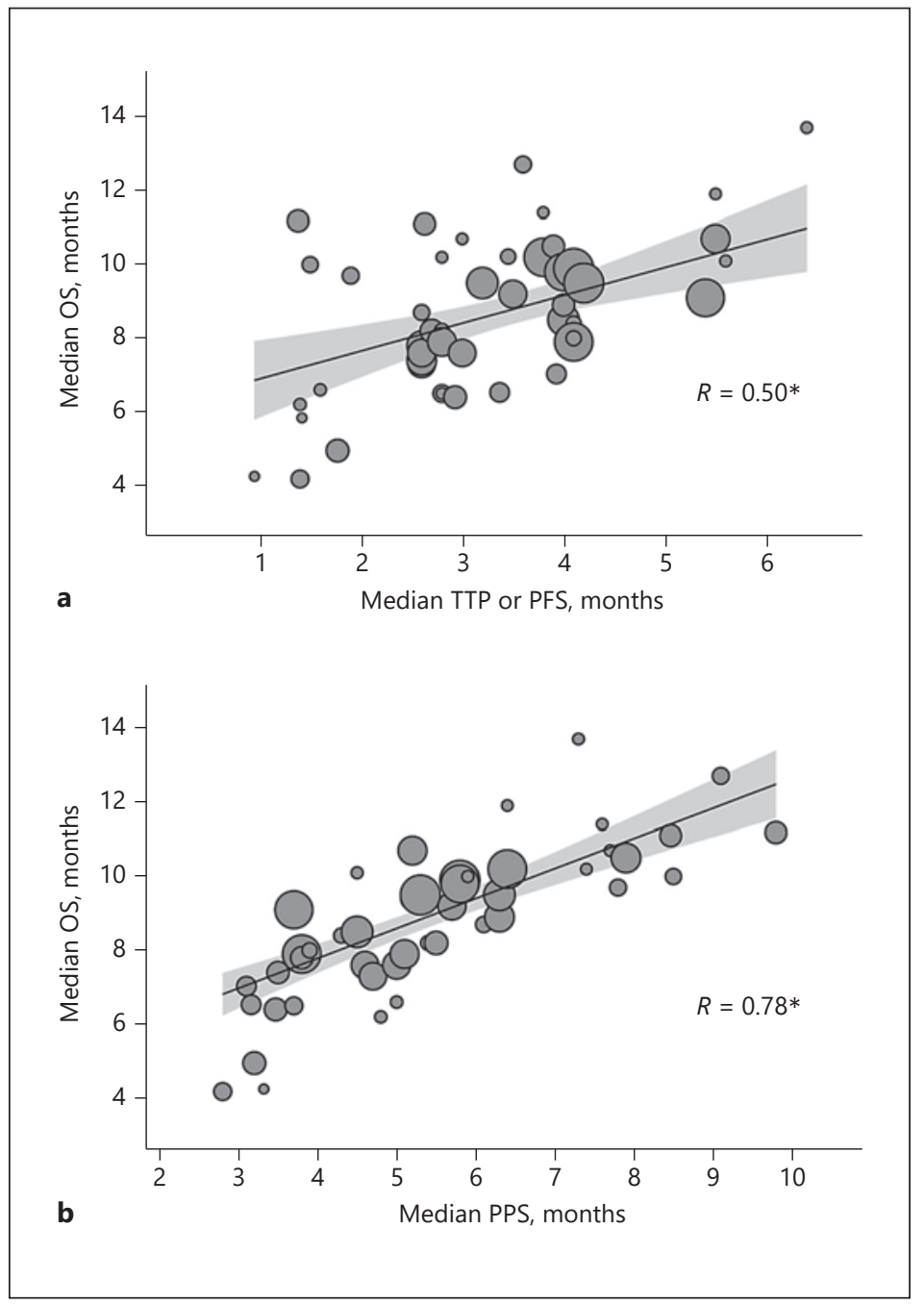

\section{Discussion}

This study investigated the correlation between TTP HR and OS HR and the correlation between mOS and either MTTP or mPPS by a systematic review and meta-analysis of randomized controlled trials of advanced HCC. Our analysis yielded three main findings: (1) TTP HR was strongly correlated with OS HR; (2) the coefficient in the regression equation between TTP HR and OS HR was not much greater than 0.48; and (3) mPPS was strongly correlated with mOS, whereas the correlation between MTTP and mOS was not so strong. These results have implications for planning and interpreting clinical trials on advanced HCC patients.

First, an important finding of this study was that the correlation between mTTP and mOS was not so strong, whereas the correlation between TTP HR and OS HR was strong. TTP is widely used as the primary endpoint in phase II trials of novel agents for unresectable solid tumors, and the judgement as to whether the agent should be tested in phase III trials and planning of such trials is generally formed based on the results of the phase II trial. However, the obtained information about OS may not have adequate power to predict a benefit from OS because the number of patients in the phase II trial is calculated based on the hypothesis 


\section{Liver Cancer}

\begin{tabular}{|c|c|}
\hline \multicolumn{2}{|l|}{ Liver Cancer 2019;8:130-139 } \\
\hline DOI: 10.1159/000489505 & $\begin{array}{l}\text { (c) } 2018 \text { S. Karger AG, Basel } \\
\text { www.karger.com/lic }\end{array}$ \\
\hline
\end{tabular}

Terashima et al.: TTP and Endpoint in Advanced HCC

regarding the expected TTP. Our data, showing a not so strong correlation between MTTP and mOS, suggest that the wrong decision may be made if planning is based only on information about mTTP. In contrast, in our data the correlation between TTP HR and OS HR was strong, indicating that it is possible to predict the OS HR from data on the TTP HR. Based on these findings, it is essential to have a control arm in order to obtain information about the HR when designing a phase II trial, since sufficient information for successful planning of a consequent pivotal study cannot be obtained from a single-arm trial that provides only median survival data [23]. Moreover, the decision as to whether a novel agent should proceed to be tested in a pivotal trial should be made based on previous results showing that the agent does not merely provide a statistical improvement in antitumor effect but has a clinically significant antitumor effect contributing to an improvement in patient outcome for efficient and successful development, all of which can be evaluated via HRs provided from an early-phase trial of a randomized design.

Second, with regard to the rationale of clinical trials in advanced HCC it is very important to note that the line of the regression equation in this study did not correspond to the line representing TTP HR equal to OS HR (shown as a dotted line in Fig. 1), and that the coefficient in the regression equation between TTP HR and OS HR was only 0.48; the risk reduction in TTP HR directly reflects the risk reduction in OS HR if the TTP HR corresponds to the OS HR and the coefficient is 1.0. OS is an indicator for a patient's prognosis, whereas TTP represents a direct clinical benefit, and whether TTP can be a surrogate for clinical benefit depends on the magnitude of the effect and the risk benefit of the new treatment compared to available therapies [24]. Our results suggest that the antitumor effect obtained from a treatment contributes only partially to the improvement in patient prognosis in advanced HCC. This tendency can be explained by our findings that mPPS strongly correlated with mOS [25]. In patients with advanced-stage HCC, locoregional therapies such as hepatic arterial infusion chemotherapy or transarterial chemoembolization have the potential to be efficacious even after sorafenib therapy [26-28], although such therapies have not been verified in proper prospective trials.

Lee et al. [29] concluded that TTP can be a surrogate endpoint for OS based only on the finding of a good correlation between TTP (or PFS) HR and OS HR; however, it is important to consider the objective of a clinical trial in addition to the correlation between TTP HR and OS HR when we evaluate the applicability of using TTP as a surrogate marker for OS. On one hand, we can certainly predict the OS HR from data on the TTP HR, as described above for an early-stage trial with the aim of judging as to whether there is value in proceeding to a pivotal trial. On the other hand, it is not appropriate to evaluate TTP as a surrogate endpoint of OS in a pivotal trial if the trial aims to verify the survival benefit from novel agents, because the contribution of the antitumor effect to the survival benefit is weakened by the strong impact of PPS on OS.

Although the US Food and Drug Administration or European Medicines Agency have usually requested proof of improvement in survival in pivotal trials for the regulatory approval of new agents [24], an increasing number of agents have been approved based on the results of a trial setting in which an indicator such as TTP directly represents the antitumor effect. This trend has often been seen for cancers such as breast cancer, colorectal cancer, or nonsmall cell lung cancer, for which several effective agents are available and it seems to be difficult to evaluate the survival benefit in a clinical trial due to the noise of PPS [30-32]. As it is well known that TTP has several disadvantages as an endpoint in this setting compared with OS [24], it is particularly important to verify this relationship and reconsider whether we can evaluate the improvement of survival prolongation in pivotal trials of advanced HCC in which the antitumor effect does not directly reflect the patients' prognosis [33]. It should originally be the antitumor effect that is evaluated in any development study of a novel agent. 


\section{Liver
Cancer}

\begin{tabular}{l|l}
\hline Liver Cancer 2019;8:130-139 \\
\hline DOI: 10.1159/000489505 & $\begin{array}{l}\text { @ 2018 S. Karger AG, Basel } \\
\text { www.karger.com/lic }\end{array}$ \\
\hline
\end{tabular}

Terashima et al.: TTP and Endpoint in Advanced HCC

Now, we may have to consider setting an indicator such as TTP which directly reflects the antitumor effect as the primary endpoint in pivotal studies of HCC as well as of breast cancer, colorectal cancer, and non-small cell lung cancer.

After our analysis, the results of the RESORCE trial were published, revealing that regorafenib improved the outcome of patients with HCC who progressed on sorafenib treatment compared with placebo in a second-line setting [20]. The point plotting OS HR $(0.63)$ of this trial against TTP HR (0.44) was located under the line representing the regression equation (see online suppl. Fig. 3), indicating that the risk reduction in TTP HR contributed more to the risk reduction in OS HR compared with previous failed trials. The reasons for this successful result were widely discussed, and they include the second-line setting, which had fewer negative effects from effective posttrial treatment. The last-line setting must be one of the elements of a successful clinical trial design that enables the antitumor effect to directly improve patients' outcomes [25].

The present study has some limitations, including the lack of individual patient data and potential confounders because of the inclusion of heterogeneous trials. Further investigations including details such as subsequent therapy, liver function, and progression patterns affecting the relationship between OS and either TTP or PPS [1] will contribute to a more efficient development of novel agents.

\section{Conclusions}

In conclusion, by a systematic review and meta-analysis of randomized controlled trials of advanced HCC, we revealed that OS HR strongly correlated with TTP HR, especially in second-line trials, whereas the correlation between mOS and mTTP was not so strong, even though mPPS strongly correlated with mOS. Predicting OS HRs from data on TTP HRs is useful when considering whether to proceed to a pivotal trial based on the results of early-phase trials. OS may be unsuitable as a primary endpoint in a pivotal trial because the improvement in antitumor effect cannot fully reflect the improvement in OS due to a strong impact of PPS on OS; we should consider to set TTP as the primary endpoint in a pivotal trial where the antitumor effect should be originally evaluated.

\section{Acknowledgments}

We thank Mary Derry, PhD ELS, from Edanz Group Ltd (www.edanzediting.com/ac) for editing a draft of this manuscript, and Prof. Kenichi Yoshimura for statistical advice.

\section{Disclosure Statement}

The authors do not have any conflict of interest. 


\section{References}

1 Reig M, Rimola J, Torres F, Darnell A, Rodriguez-Lope C, Forner A, et al: Post-progression survival of patients with advanced hepatocellular carcinoma. Rationale for second line trial design. Hepatology 2013;58:20232031.

2 Chen PJ, Furuse J, Han KH, Hsu C, Lim HY, Moon H, et al: Issues and controversies of hepatocellular carcinomatargeted therapy clinical trials in Asia: experts' opinion. Liver Int 2010;30:1427-1438.

3 Lencioni R, Montal R, Torres F, Park JW, Decaens T, Raoul JL, et al: Objective response by mRECIST as a predictor and potential surrogate end-point of overall survival in advanced HCC. J Hepatol 2017;66:11661172.

4 Llovet JM, Di Bisceglie AM, Bruix J, Kramer BS, Lencioni R, Zhu AX, et al: Design and endpoints of trials in hepatocellular carcinoma. J Natl Cancer Inst 2008;100:698-711.

5 Siegel RL, Miller KD, Jemal A: Cancer statistics, 2016. CA Cancer J Clin 2016;66:7-30.

6 Llovet JM, Ricci S, Mazzaferro V, Hilgard P, Gane E, Blanc JF, et al: Sorafenib in advanced hepatocellular carcinoma. N Engl J Med 2008;359:378-390.

7 Cheng AL, Kang YK, Chen Z, Tsao CJ, Qing S, Kim JS, et al: Efficacy and safety of sorafenib in patients in the AsiaPacific region with advanced hepatocellular carcinoma: a phase III randomised, double-blind, placebocontrolled trial. Lancet Oncol 2009;10:25-34.

8 Barbare JC, Bouché O, Bonnetain F, Dahan L, Lombard-Bohas C, Faroux R, et al: Treatment of advanced hepatocellular carcinoma with long-acting octreotide: a phase III multicentre, randomised, double blind placebocontrolled study. Eur J Cancer 2009;45:1788-1797.

9 Qin S, Bai Y, Lim HY, Thongprasert S, Chao Y, Fan J, et al: Randomized, multicenter, open-label study of oxaliplatin plus fluorouracil/leucovorin versus doxorubicin as palliative chemotherapy in patients with advanced hepatocellular carcinoma from Asia. J Clin Oncol 2013;31:3501-3508.

10 Johnson PJ, Qin S, Park JW, Poon RT, Raoul JL, Phillip PA, et al: Brivanib versus sorafenib as first-line therapy in patients with unresectable, advanced hepatocellular carcinoma: results from the randomized phase III BRISK-FL study. J Clin Oncol 2013;31:3517-3524.

11 Cheng AL, Kang YK, Lin DY, Park JW, Kudo M, Qin S, et al: Sunitinib versus sorafenib in advanced hepatocellular cancer: results of a randomized phase III trial. J Clin Oncol 2013;31:4067-4075.

12 Cainap C, Qin S, Huang WT, Chung IJ, Pan H, Cheng Y, et al: Linifanib versus sorafenib in patients with advanced hepatocellular carcinoma: results of a randomized phase III trial. J Clin Oncol 2015;33:172-179.

13 Zhu AX, Rosmorduc O, Evans TR, Ross PJ, Santoro A, Carrilho FJ, et al: SEARCH: a phase III, randomized, doubleblind, placebo-controlled trial of sorafenib plus erlotinib in patients with advanced hepatocellular carcinoma. J Clin Oncol 2015;33:559-566.

14 Abou-Alfa GK, et al: Phase III randomized study of sorafenib plus doxorubicin versus sorafenib in patients with advanced hepatocellular carcinoma (HCC): CALGB 80802 (Alliance). J Clin Oncol 2016;34(suppl):192.

15 Llovet JM, Decaens T, Raoul JL, Boucher E, Kudo M, Change C, et al: Brivanib in patients with advanced hepatocellular carcinoma who were intolerant to sorafenib or for whom sorafenib failed: results from the randomized phase III BRISK-PS study. J Clin Oncol 2013;31:3509-3516.

16 Zhu AX, Kudo M, Assenat E, Cattan S, Kang YK, Lim HY, et al: Effect of everolimus on survival in advanced hepatocellular carcinoma after failure of sorafenib: the EVOLVE-1 randomized clinical trial. JAMA 2014;312:57-67.

17 Zhu AX, Park JO, Ryoo BY, Yen CJ, Poon R, Pastorelli D, et al: Ramucirumab versus placebo as second-line treatment in patients with advanced hepatocellular carcinoma following first-line therapy with sorafenib (REACH): a randomised, double-blind, multicentre, phase 3 trial. Lancet Oncol 2015;16:859-870.

18 Kudo M, Moriguchi M, Numtata K, Hidaka H, Tanaka H, Ikeda M, et al: S-1 versus placebo in patients with sorafenib-refractory advanced hepatocellular carcinoma (S-CUBE): a randomised, double-blind, multicentre, phase 3 trial. Lancet Gastroenterol Hepatol 2017;2:407-417.

19 Ghassan K, et al: Phase III randomized study of second line ADI-peg 20 (A) plus best supportive care versus placebo (P) plus best supportive care in patients (pts) with advanced hepatocellular carcinoma (HCC). J Clin Oncol 2016;34(suppl):4017.

20 Bruix J, Qin S, Merle P, Granito A, Huang YH, Bodoky G, et al: Regorafenib for patients with hepatocellular carcinoma who progressed on sorafenib treatment (RESORCE): a randomised, double-blind, placebocontrolled, phase 3 trial. Lancet 2017;389:56-66.

21 Terashima T, Yamashita T, Takata N, Nakagawa H, Toyama T, Arai K, et al: Post-progression survival and progression-free survival in patients with advanced hepatocellular carcinoma treated by sorafenib. Hepatol Res 2016;46:650-656.

22 Sargent DJ, Wieand HS, Haller DG, Gray R, Benedetti JK, Buyse M, et al: Disease-free survival versus overall survival as a primary end point for adjuvant colon cancer studies: individual patient data from 20,898 patients on 18 randomized trials. J Clin Oncol 2005;23:8664-8670.

23 Llovet JM, Hernandez-Gea V: Hepatocellular carcinoma: reasons for phase III failure and novel perspectives on trial design. Clin Cancer Res 2014;20:2072-2079.

24 US Department of Health and Human Services: Guidance for Industry: Clinical Trial Endpoints for the Approval of Cancer Drugs and Biologics. US Department of Health and Human Services, 2007. https://www.fda.gov/ downloads/Drugs/GuidanceComplianceRegulatoryInformation/Guidances/ucm071590.pdf. 
25 Morita S, Sakamaki K, Ying G: Detecting overall survival benefit derived from survival postprogression rather than progression-free survival. J Natl Cancer Inst 2015;107:djv133.

26 Kudo M: Regorafenib as second-line systemic therapy may change the treatment strategy and management paradigm for hepatocellular carcinoma. Liver Cancer 2016;5:235-244.

27 Terashima T, Yamashita T, Arai K, Sunagozaka H, Kitahara M, Nakagawa H, et al: Feasibility and efficacy of hepatic arterial infusion chemotherapy for advanced hepatocellular carcinoma after sorafenib. Hepatol Res 2014;44:1179-1185.

28 Terashima T, Yamashita T, Horii R, Arai K, Kawaguchi K, Kitamura K, et al: Potential efficacy of therapies targeting intrahepatic lesions after sorafenib treatment of patients with hepatocellular carcinoma. BMC Cancer 2016;16:338.

29 Lee DW, Jang MJ, Lee KH, Cho EJ, Lee JH, Yu SJ, et al: TTP as a surrogate endpoint in advanced hepatocellular carcinoma treated with molecular targeted therapy: meta-analysis of randomised controlled trials. Br J Cancer 2016;115:1201-1205.

30 Hayashi H, Okamoto I, Morita S, Taguri M, Nakagawa K: Postprogression survival for first-line chemotherapy of patients with advanced non-small-cell lung cancer. Ann Oncol 2012;23:1537-1541.

31 Petrelli F, Barni S: Correlation of progression-free and post-progression survival with overall survival in advanced colorectal cancer. Ann Oncol 2013;24:186-192.

32 Saad ED, Katz A, Buyse M: Overall survival and post-progression survival in advanced breast cancer: a review of recent randomized clinical trials. J Clin Oncol 2010;28:1958-1962.

33 Ocana A, Tannock IF: When are "positive" clinical trials in oncology truly positive? J Natl Cancer Inst 2011;103: $16-20$. 